\title{
Elementos que fundamentan el Acceso Abierto
}

\author{
Adolfo Rodríguez Gallardo *
}

Artículo recibido:

14 de diciembre de 2006.

Artículo aceptado:

16 de noviembre de 2007.

\section{RESUMEN}

Las bibliotecas han soportado durante más de dos décadas el constante incremento habido en el costo de las publicaciones periódicas que ponen a disposición de sus comunidades. No se trata sólo de una cuestión financiera, el trasfondo es una grave interferencia en la comunicación científica que ha sobrepasado la esfera del trabajo bibliotecario.

El costo de las suscripciones de la comunicación científica, ha sido analizado por aquellos a quienes afecta más significativamente: científicos y académicos, quienes han concluido que la solución al problema son las publicaciones científicas de acceso libre. Se intenta determinar que los principales factores que fundamentan

* Centro Universitario de Investigaciones Bibliotecológicas de la UNAM, México. jadolfo@servidor.unam.mx

INVESTIGACIÓN BIBLIOTECOLÓGICA, Vol. 22, Núm. 44, enero/abril, 2008, México, ISSN: 0187-358X. pp. 161-182 
el paradigma del acceso abierto identificando los intereses que se mueven alrededor de este modelo y de la publicación comercial.

Se encontró que las iniciativas, declaraciones y proyectos del acceso abierto favorecen el ingreso irrestricto a la información por parte de la comunidad científica y se analizan y argumentan, con relación al costo de la información, las restricciones derivadas del derecho de autor, la calidad de la información, y los aspectos tecnológicos entre los que se encuentran la interoperatividad y la normalización. También se identifican acciones concretas de algunas de las publicaciones de acceso libre que han sido puestas en marcha.

Palabras clave: Publicaciones de libre acceso; Comunicación científica; Suscripciones; Bibliotecas académicas.

\section{ABSTRACT}

\section{Foundational elements for Open Access} Adolfo Rodríguez Gallardo

For more than two decades libraries have suffered a constant cost increase in periodicals available to their readers. However it is not just a matter of financing, a considerable interference from the scientific communication and has already overtaken the librarian.

Scientific communication and subscription costs have been analysed by those who are most affected, namely scientists and academics, who have concluded that the solution to this problem is free access to scientific publications. The main arguments for Open Access are presented, together with interests associated with this model and with commercial publishing.

It was found that the initiatives, declarations and projects of Open Access favor the unrestricted access to information by the scientific community.

Keywords: Open Access publications; Scientific communication; Subscription to periodicals; Academic libraries. 


\section{INTRODUCCIÓN}

A finales de la década de los años 1970, Richard De Gennaro señalaba que A el costo de las suscripciones se había convertido en un monstruo que engullía el presupuesto de las bibliotecas. El artículo "Escalating journal prices: time to fight back" (El aumento en los precios de las revistas: tiempo de defenderse) de De Gennaro fue el tema que ilustró la cubierta de American Libraries en febrero de 1977, y la imagen elegida fue la de un Journal-King Kong en la cima del edificio Biblioteca-Empire State llevándose cautiva a la frágil Dama-Presupuesto en una mano.

Es un hecho que los costos de las suscripciones han aumentado en forma sostenida durante los últimos 20 años, aun por encima de los índices de inflación mundial y local, mientras que los presupuestos de las bibliotecas se han mantenido sin cambio o han experimentado incrementos marginales muy por debajo del aumento en los costos de las adquisiciones y la operación de las bibliotecas. Tal situación ha producido que éstas busquen formas de disminuir sus gastos recurriendo a la supresión o limitación de recursos o servicios.

Con la generalización de Internet en la década de los años noventa, se pensó que se había encontrado el instrumento que permitiría ampliar la accesibilidad de las revistas y abaratar su costo. Hubo editores que pusieron en línea algunos de sus títulos y por no contar con un esquema de cobro permitieron que éstos se consultaran sin cargo alguno en las versiones conocidas como texto completo, por lo que se pensó que la tendencia sería la gratuidad.

En sus principios, la consulta de las publicaciones periódicas a través de Internet demostró no ser cien por ciento confiable por motivos técnicos, así que las bibliotecas encararon dos opciones: alojar las revistas de texto completo en sus equipos, o realizar la consulta en línea, que era, por decir lo menos, errática. Independientemente de la decisión que se tomara, con el tiempo la comunidad bibliotecaria se percató de que los editores no abandonarían la publicación en papel por la publicación electrónica sino que las combinarían obligando a las bibliotecas a pagar un cargo adicional por las versiones en línea, por lo que éstas continuaron haciendo importantes esfuerzos para no suspender sus suscripciones.

Esta situación ha preocupado a un amplio segmento de la comunidad académica vinculado con los procesos de edición de publicaciones periódicas, con su comercialización y distribución, y con las dificultades que se presentan para hacerlas accesibles a los miembros de sus comunidades.

Las bibliotecas intentaron resolver este problema formando consorcios para adquirir sus suscripciones en forma conjunta, mientras que un importante 
grupo de autores, dirigentes universitarios, instituciones de investigación y bibliotecarios plantearon la posibilidad de poder acceder a los productos de la investigación sin cargo para los lectores o usuarios, y la necesidad y conveniencia de buscar nuevas formas de financiar su publicación.

Este planteamiento se ha denominado acceso abierto y constituye el objeto de este trabajo, que busca determinar los principales factores que dan origen al paradigma de las publicaciones de libre acceso, e identificar los intereses de los sectores involucrados, así como reconocer las coincidencias que puedan existir entre las diversas iniciativas y proyectos.

\section{INICIATIVAS SOBRE EL LIBRE ACCESO A LA INFORMACIÓN CIENTÍFICA Y/O ACADÉMICA}

A lo largo del siglo pasado la comunicación científica se ha visto ampliamente influida por la tecnología y las tendencias que predominan en el mercado internacional de la información. En este sentido el costo de la información ha motivado que la comunidad científica y académica se cuestione por qué debe pagar para acceder sin restricciones a la información que ella misma genera.

El modelo de acceso abierto surge entonces como la propuesta más discutida en la actualidad para hacer frente a los altos costos de la información y para facilitar la comunicación científica; modelo que no ha sido fácil de explicar y mucho menos de aceptar dados los diferentes intereses que se mueven en el campo de las publicaciones científicas, entre los que se encuentran las ganancias de los editores, la calidad académica de la producción científica, el prestigio profesional y los intereses de los autores. El acceso abierto ha generado puntos de vista distintos y en ocasiones encontrados que se discuten con cierta pasión entre bibliotecarios, académicos, intermediarios y editores (Corrado, 2005, 1)

Para tratar de explicar qué es el acceso abierto y cuáles son sus características nos ayudaremos de la tabla 1 (página 73), que resume las iniciativas generales surgidas de la participación conjunta de sociedades y organizaciones científicas, universidades, editores, científicos y bibliotecarios.

Otras declaraciones y proyectos que apoyan el acceso libre a la información se han generado al interior de otras organizaciones entre las que se encuentran las incluidas en la tabla 2 (página 74). 


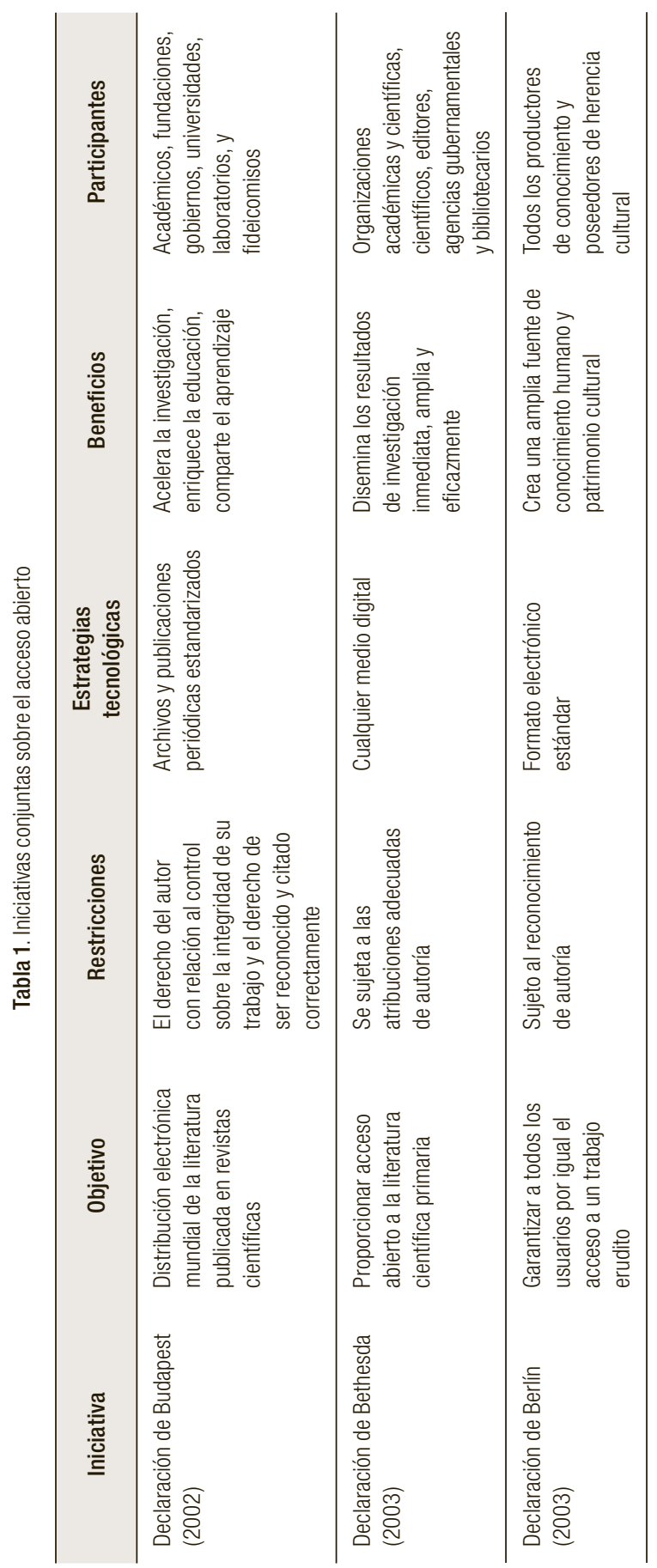




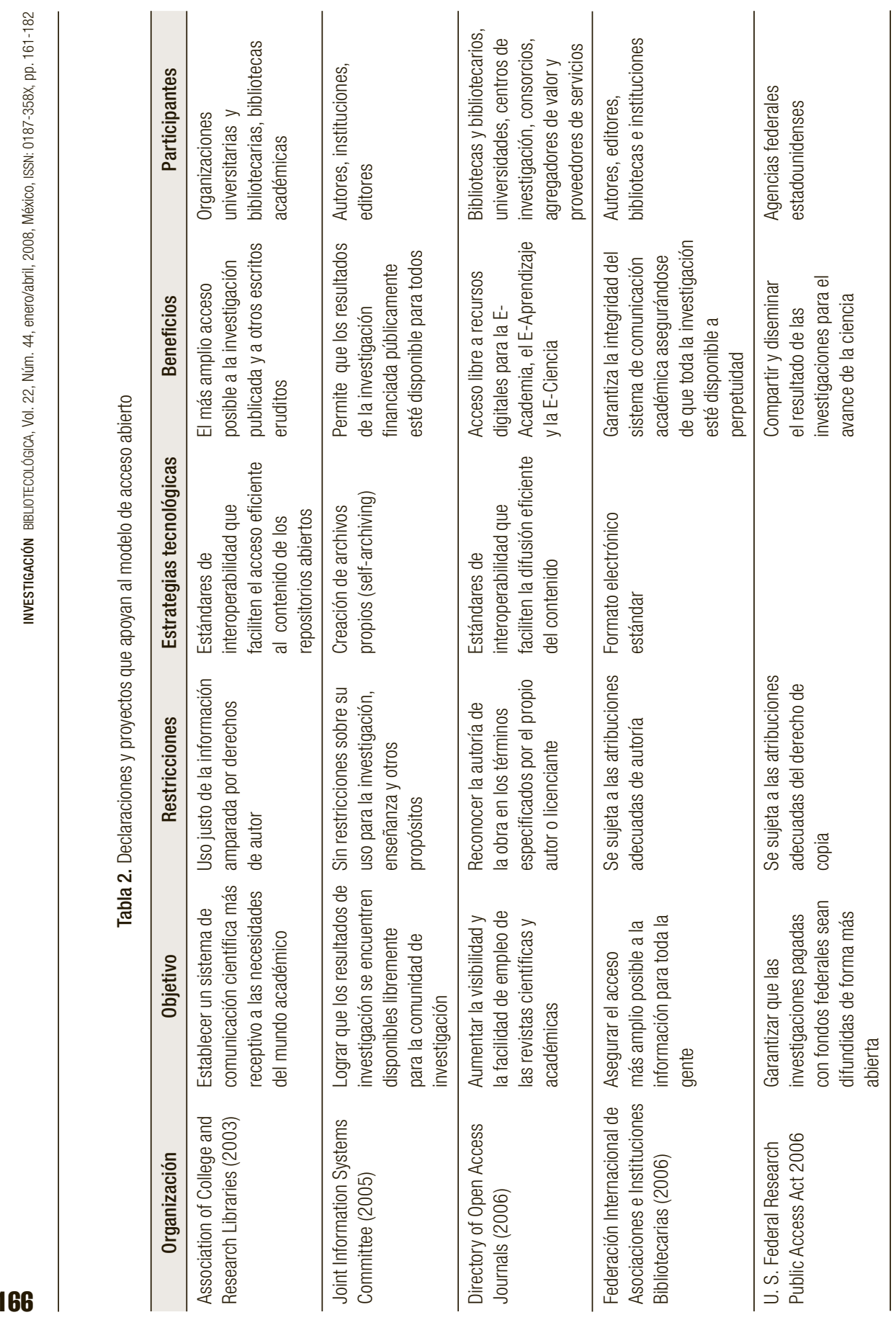


El objetivo general de iniciativas y proyectos es optimizar el valor y la calidad de la investigación, permitirle a los investigadores el acceso a información académica y/o científica bajo mínimas exigencias a través de publicaciones de libre acceso. Los objetivos de interés particular están determinados por los campos de acción de los involucrados, pero también entre ellos existen coincidencias.

$\mathrm{El}$ acceso abierto adopta diferentes manifestaciones al mismo tiempo que mantiene los aspectos fundamentales que le dan identidad, Willinsky (2003, 264-265) ha identificado nueve diferentes formas en que se puede presentar este tipo de acceso:

1) Servidores de impresos electrónicos (eprints-servers): los autores guardan sus pre o post impresos en archivos de acceso abierto;

2) Acceso abierto ilimitado: publicación inmediata, completa y exclusivamente de acceso abierto;

3) Acceso abierto bimodal: ofrece el libre acceso y la versión impresa de una publicación;

4) Acceso abierto aplazado: la publicación se encuentra en acceso libre sólo después de seis meses de haber sido publicada para los suscriptores;

5) Acceso abierto pagado por el autor: el autor cubre los costos generados por publicar en el modelo de acceso abierto;

6) Acceso abierto parcial: sólo algunos artículos de una revista están disponibles en acceso abierto;

7) Acceso abierto per-cápita: el acceso abierto está disponible basado en el ingreso per cápita de los habitantes de un país;

8) Acceso abierto a resúmenes o abstracts: se permite el acceso abierto sólo a tablas de contenido y resúmenes; y

9) Acceso abierto cooperativo: los miembros institucionales cooperan y apoyan el acceso abierto de las publicaciones periódicas.

Suber (2002, [3.1]) señala que el acceso abierto significa la posibilidad de llegar al texto sin ningún costo para los usuarios o para las bibliotecas, lo cual se hace para acelerar la investigación y compartir el conocimiento; en este esquema los editores buscan que los costos sean cubiertos por otras fuentes.

La Federal Research Public Access Act of 2006 (FRPAA) de los Estados Unidos pretende tomar una serie de medidas para garantizar que las investigaciones pagadas con fondos federales sean difundidas de forma más abierta. La medida más importante es la que señala que seis meses después de que una investigación haya sido publicada en una revista que cuente con evaluación de pares, debe estar a la disposición de todas las personas en un archivo abierto. 
El proyecto ha generado gran cantidad de respuestas tanto de asociaciones académicas como de la asociación de editores la cual se opone a esta medida, por considerar que puede afectar desfavorablemente a la investigación; hay quienes opinan que obligar a los autores a publicar los resultados de sus investigaciones en forma abierta genera conflicto con los derechos de autor.

Para apoyar este proyecto de ley 25 altos funcionarios de universidades públicas y privadas (prebostes) han publicado el documento An Open Letter to the Higher Education Community (Carta Abierta a la Comunidad de Educación Superior) en la que dejan en claro que los intereses de las comunidades académicas no son los mismos que los de las grandes editoriales, por lo que señalan que:

Una amplia diseminación de los resultados de la investigación académica es esencial y adecuada para lograr el compromiso de avanzar y difundir el conocimiento y por lo tanto es buena para la educación y la investigación... Por lo tanto, apoyamos la premisa básica de la propuesta S2695 que permite un acceso más amplio a las nuevas ideas que sean producto de investigaciones apoyadas con fondos gubernamentales, esto promueve el progreso, el crecimiento económico y el bienestar público (An Open Letter 2006, 1).

Jaschik $(2006,1)$ sostiene que el apoyo que las universidades implicadas le brindan a la FRPAA surge del siguiente cuestionamiento:

Si son las universidades las que pagan los sueldos de los investigadores y los proveen de laboratorios, y el gobierno federal les otorga financiamiento a aquellas ¿por qué tienen la sensación de que no pueden tener acceso a los resultados de la investigación?

Los prebostes se manifestaron en el sentido en que sus necesidades como comunidades de eruditos no son los mismos que las de los grandes editores comerciales; al respecto Suber $(2006,2)$ añade que debido a que los eruditos no ganan dinero por publicar sus artículos, su posición es muy diferente, por ejemplo, a la de los músicos y de quienes hacen películas.

Son muchas las variables que pueden influir en el éxito o fracaso del acceso abierto, sin embargo es importante mantener en mente que aunque se expanda su paradigma, habrá por lo menos cuatro barreras que impedirán el acceso a la literatura:

1. Barreras de filtrado y censura. Muchas escuelas, empleadores y gobiernos desean limitar lo que usted puede ver. 
2. Barreras lingüísticas. La mayoría de la literatura en línea está en inglés, o apenas en una lengua, y la traducción automática es muy mala.

3. Barreras de acceso para incapacitados. La mayoría de los sitios de web todavía no son tan accesibles para los usuarios que tienen alguna limitación.

4. Barreras en la conectividad. La brecha digital dejaría a billones de personas, incluyendo a millones de eruditos, fuera de línea (Suber 2006, 7).

\section{El costo de la información y el acceso abierto}

Desde un punto de vista económico, Anderson $(2004,1)$ ha dicho atinadamente que la creencia de que la información es gratuita es un mito, no hay información gratuita porque producirla siempre tiene un costo. Si bien es cierto que la producción académica de una persona puede incluirse en el salario que recibe de una institución, también lo es que el costo de tal información es cubierto por la institución en la que trabaja el académico que la produce. Las ideas pueden ser libres, pero éstas no se transforman en información hasta que una persona crea una representación simbólica de ellas; subir esa representación a la red, mantenerla y conservarla tiene un costo que alguien ha de cubrir.

Los académicos, quienes normalmente reciben un salario por crear información, intercambian sus productos por varias cosas, entre las que no está el derecho de autor, o al menos no de forma prioritaria. Para ellos es más importante el crédito que obtienen al publicar en editoriales o en revistas de reconocido prestigio entre sus pares (Anderson 2004, 2).

Al analizar los costos de la información se ha llegado a pensar que una institución paga dos veces por un artículo: una vez para producirlo y otra para consultarlo, es por ello que la iniciativa de acceso abierto se ha visto como una alternativa para frenar el incremento de precios (Corrado 2005, 2). En este contexto el acceso abierto carecería de sentido si no se le considera como una forma de ayudar a controlar el costo de las publicaciones periódicas y, si es posible, de disminuir el gasto que las bibliotecas hacen para ofrecerles a sus usuarios acceso a las publicaciones periódicas científicas y humanísticas que requieren para su trabajo académico (Goodman 2004, 2).

Se trata principalmente, aunque no de manera exclusiva, de facilitar el acceso a documentos en los que se presentan los resultados de experimentos, descubrimientos y análisis novedosos sobre un tema. Si la aseveración sobre el doble pago fuera cierta, el modelo de producción, bajo la iniciativa del acceso abierto, tomaría las siguientes vertientes: 
a) Si la institución paga el salario del investigador y asume también los gastos de la publicación sea ésta en papel o electrónica (mantenimiento del servidor, etcétera) está invirtiendo dos veces;

b) Si la institución que le paga al investigador no asume los gastos de la publicación será el autor quien pague por ella y retenga sus derechos. La institución y el autor pagan;

c) Si la institución paga el salario del investigador y éste publica en revistas de acceso abierto en las que el autor no paga por publicar, sólo la institución es la que paga;

d) La comunidad de una institución que no pagó el salario del investigador tiene acceso al artículo de éste sin ningún cargo, pero sin embargo habrá pagado por sus propios investigadores y publicaciones.

Bacher (Jaschik 2006, 4) y Suber $(2004,1)$ afirman que la literatura de acceso abierto no se produce sin hacer erogaciones, a pesar de que sus costos de producción sean menores que los de una publicación convencional. Se reconocen diversas formas de acceso abierto, por lo que Bacher sugiere que sean las prensas universitarias las que se conviertan en participantes activos de esta iniciativa.

Antes del modelo de acceso abierto, tradicionalmente la institución pagaba el salario del investigador y compraba una suscripción a la revista en la que éste publicaba; la institución invertía dos veces. Melero $(2005,255)$ y Suber $(2004,1)$ coinciden en que bajo el nuevo paradigma la inversión debe ir encaminada a favorecer la difusión de la información y no a crear barreras de acceso a ésta como estaba sucediendo.

En este sentido Suber $(2006,6)$ considera que el acceso abierto busca crear en lugar de destruir al señalar lo siguiente:

Aunque el precio de las revistas se haya incrementado cuatro veces más rápido que la inflación, desde mediados de los años ochenta, el propósito del acceso abierto no es castigar o minar su publicación, sino proporcionar una alternativa accesible y tomar ventaja de la nueva tecnología a Internet para tener una distribución más amplia al mismo tiempo que reducir sus costos. Por otra parte, la motivación de los investigadores no es solucionar la crisis de tasación de las publicaciones sino ampliar el número y facilitar el acceso de los lectores; en otros términos llegar a un público más grande y maximizar su impacto.

Anderson $(2004,2)$ aborda también el mito que considera que la información es un bien público; en este caso argumenta que el hecho de que la información sea buena para la gente o que su amplia distribución sea benéfica para 
el público en general, no la convierte en un bien público, pues la información forma parte de la propiedad intelectual de aquellos que la crean. Además la propiedad intelectual está protegida por un instrumento legal que le garantiza a los productores el derecho sobre sus creaciones.

Plantear la consulta gratuita no significa que la producción de las publicaciones periódicas de acceso abierto no involucre costos. En este sentido se ha comprobado que los recursos empleados en la diseminación electrónica de las publicaciones son menores que aquellos invertidos en las formas convencionales de diseminación y almacenamiento. Un sistema de acceso libre requiere del cálculo cuidadoso de los costos de recuperación y financiamiento, pero la reducción de los gastos que se observa hasta ahora hace pensar que la posibilidad de tener archivos de publicaciones periódicas de alta calidad bajo este esquema no es meramente una utopía. Por ello el acceso abierto representa una buena oportunidad para que las instituciones y personas interesadas en la diseminación del conocimiento no paguen los altos costos comerciales, como ha venido ocurriendo.

Si bien el Joint Information Systems Committee (JISC 2005,1) declara que el acceso abierto no es la ruta para acotar los altos costos de la publicación, la declaración de Budapest (BOAI 2002) propone que aquellos que apoyen el libre acceso no deberán cobrar suscripciones ni cuotas de acceso a la información, y la declaración de Bethesda $(2003,2-5)$ asume que los costos de publicación deben ser considerados entre los costos de la investigación, pues la diseminación de los resultados es parte importante de ésta.

Mediante la puesta en marcha de algunas opciones de acceso abierto se ha podido concluir que económicamente es posible su realización, y ésta que le proporciona al lector un poder extraordinario para localizar y hacer uso de literatura relevante, además de que les ofrece a los autores y a sus trabajos una vasta y mesurable nueva visibilidad, que les asegura serán leídos y que sus trabajos tendrán impacto.

\section{Tecnología y acceso abierto}

El Acceso Abierto a la literatura científica y académica requiere que ésta sea digital, en línea, gratuita y que esté libre de la mayoría de las restricciones que imponen el copyright y las licencias (Suber 2004, 1). En este contexto la eficiencia de los equipos de cómputo y su interconectividad, así como la existencia de formatos estándares en los que se almacene la información, como el PDF y HTML, es fundamental.

El acceso a la información científica y académica se verá limitada solamente por las barreras inherentes al acceso en la Internet pública, establece 
la Declaración de Budapest. Contar con acceso libre a un creciente número de publicaciones periódicas se logrará a través de la conformación de autoarchivos y de una nueva generación de publicaciones.

El autoarchivo se crea cuando el autor de un trabajo lo deposita, al menos, en un repositorio en línea en un archivo electrónico elaborado con los estándares creados para tal efecto, con lo cual permite que los motores de búsqueda y otros instrumentos lo recuperen sin que el usuario necesariamente sepa en qué lugar se aloja aquél.

La nueva generación de publicaciones, entiéndase como publicaciones electrónicas, no deberá restringir su acceso y uso apelando a la protección de los derechos de autor sino que se comprometerá a promover el acceso libre, la distribución irrestricta, la interoperabilidad y la preservación de la información científica y académica que contiene.

Triska y Café (2001) destacan los aspectos que deben ser tomados en cuenta cuando se trata de la creación de archivos abiertos y de la compatibilidad operativa:

\section{Compatibilidad}

- Definición de un conjunto mínimo de datos.

- Concordancia en el uso de una sintaxis común XML, para transportar y representar los datos.

- Definición de un protocolo común para extraer datos.

\section{Creación de repositorios}

- Mecanismos de presentación de los trabajos.

- Sistema de almacenamiento a largo plazo.

- Políticas de gestión, que tomen en cuenta las normas de publicación y preservación digital.

- Interface abierta, que permita reunir datos.

- Uso del software de fuente abierta (open source).

Auque técnicamente sea factible implementar el modelo de libre acceso, también se debe considerar que para llevarlo a cabo es necesario tomar en cuenta el tamaño del mercado de la información, porque algunos sectores de éste no tienen, por el momento, capacidad para desarrollar la infraestructura que sería necesaria. 


\section{Publicaciones periódicas electrónicas y acceso abierto}

El Directory of Open Access Journals (Directorio de Publicaciones Periódicas de Acceso Libre, http://www.doaj.org) ilustra uno de los esfuerzos realizados para proveer acceso a publicaciones de calidad. En él se incluyen 2,285 títulos de publicaciones periódicas, de las que 651 se pueden acceder a nivel de artículo, y se cuenta actualmente con 101,248 artículos; también incluye un listado temático y el número de títulos por cada tema. Las obras incluidas en este directorio se ajustan a la declaración de Budapest: pretenden ser publicaciones que contienen artículos de alta calidad, lo que garantizan mediante su evaluación por pares, y recoger trabajos de investigación lo que les permite ser evaluados como una publicación de investigación.

El Directory of Open Access Journals define una publicación periódica de acceso libre como aquella que "usa un modelo de financiamiento que le permite no cobrarles a los lectores o a sus instituciones por el acceso", y en apego a la Declaración de Budapest acepta el derecho de los usuarios a "leer, bajar, copiar, distribuir, imprimir, buscar, o ligar el texto completo de estos artículos" (DOAJ, 2006, 2). El directorio asume que las publicaciones periódicas de acceso libre deben ser evaluadas por pares o incluir un control de calidad editorial, y las principales iniciativas insisten en su importancia (Suber 2004, 1); respecto de su contenido se comprometen a incluir principalmente los resultados de investigaciones o avances de las mismas para una comunidad académica.

Las publicaciones que son enteramente de acceso abierto, conocidas como Golden OA, son aquellas cuyos costos son cubiertos por los autores o las instituciones que los patrocinan (Goodman 2004, 5). No obstante, Jaschik $(2006,2)$ señala:

Las asociaciones académicas (muchas de las cuales dependen para sus presupuestos de las ventas de sus publicaciones) se han manifestado en contra del acceso abierto, aun cuando sus miembros exigen cada vez más el acceso libre y en línea, de la información.

Si las virtudes que poseen las publicaciones en papel tienen que ser sacrificadas en aras del acceso abierto será difícil defender éste, pero tal sacrificio no es necesario. Suber (2002, [3.1]) presenta una magnífica síntesis de las principales ventajas que tienen las publicaciones periódicas tradicionales publicadas en papel, las que, asegura, no tienen por que eliminarse cuando se producen publicaciones de acceso abierto. Estas ventajas son ocho y se resumen a continuación: 
- Evaluación de pares. Las principales iniciativas de acceso abierto están de acuerdo en que la evaluación de pares es esencial para las publicaciones de este tipo, las publicaciones impresas en papel, o las que tienen un costo de suscripción. El libre acceso pretende quitar las barreras de precio, no los filtros de control de calidad.

- Calidad profesional. La calidad de las publicaciones está en relación con la calidad de los editores, evaluadores y autores. Estos tres elementos son independientes del costo de adquisición de las publicaciones (con costo o gratuitas) y del medio de entrega (impreso o electrónico).

- Prestigio. Prestigio no es lo mismo que calidad. Si la calidad es en verdad muy buena, entonces el prestigio es reconocido como excelente. Puesto de esta forma parece que la calidad fuera más importante que el prestigio. Pero el incentivo de los autores para someter un trabajo a la consideración de una publicación o editorial está más relacionado con el prestigio de ésta que con su calidad, es ahí donde los dos difieren.

- Preservación. Hasta el momento el medio más usado para conservar los textos por cientos de años ha sido el papel. La preservación digital deberá ser tan buena como lo fue el papel hasta antes de la era digital.

- Propiedad intelectual. El acceso abierto es compatible con los derechos de autor en tanto los titulares de tales derechos estén de acuerdo con el acceso abierto. El derecho de autor le da certidumbre a éste de que las copias autorizadas no se mutilen o se "pirateen". El hecho de que el titular de los derechos de autor consienta en el acceso libre distingue a este acceso de lo que puede llamarse Napster para las Ciencias.

- Ganancias. La publicación de acceso abierto es compatible con el crédito, e incluso con las ganancias, tal como es compatible con los modelos de las asociaciones sin fines de lucro. Las ganancias de una revista de acceso abierto no pueden provenir de las suscripciones o licencias, ya que eso viola la naturaleza libre de barreras que caracteriza al acceso abierto. En lugar de cobrarles a los lectores por el acceso o a sus agrupaciones hay que cobrarles a los autores o a sus patrocinadores por la diseminación; por tanto el ingreso consiste en las cuotas por diseminación, más la venta de anuncios y servicios auxiliares.

- Cobro por anuncios. Una revista con acceso abierto les ofrece a los lectores la posibilidad de acceder a literatura esencial sin ningún cobro. Una publicación científica puede vender anuncios y servicios auxiliares como alertas de información o el servicio de liga a otros materiales de la misma revista.

- Edición impresa. El acceso abierto se refiere al acceso libre en línea pero es perfectamente compatible con otras clases de acceso al mismo 
contenido. El editor de una revista de acceso abierto puede perder dinero si produce una versión impresa, y por eso algunos editores han decidido no hacerlo, pero un editor puede decidir vender la edición impresa al costo para aquellos que la necesiten. Por supuesto, la impresión en papel es compatible con la versión en línea cuando lo que se busca es la preservación a largo plazo.

La diseminación efectiva y eficiente de los resultados de la investigación es esencial para el avance del conocimiento. Por ello, las publicaciones de acceso libre también deben ser difundidas ampliamente entre la comunidad y preservadas adecuadamente. En este aspecto la participación de bibliotecarios y bibliotecas se considera ineludible, éstos deberán incluir y destacar en sus catálogos y bases de datos las publicaciones de acceso abierto, pues de nada serviría el acceso irrestricto a la información si no hubiera alguien que la usara.

\section{Derechos de autor y acceso abierto}

Eliminar las barreras de acceso a la información es un punto polémico en el modelo de acceso abierto. Si bien es cierto que las iniciativas coinciden en que el acceso a la información científica y académica debe ser irrestricta, para que los usuarios la usen con cualquier propósito, también lo es que reconocen el derecho de atribución de la autoría y el derecho de los autores a mantener la integridad de su trabajo, este planteamiento suena radical desde el punto de vista de la publicación convencional. En ésta los derechos se constriñen al uso justo, que es muy difícil de determinar, a las licencias restrictivas y a los costos de las licencias.

A medida que las revistas pasan de la forma impresa a la electrónica, el marco legal sobre su uso cambia de la ley de propiedad intelectual (copyright) hacia la ley contractual (ACRL 2003,156).

Actualmente las bibliotecas tienen que suscribir contratos o convenios con los editores con quienes se establece lo que les está permitido realizar en cuanto a préstamo, así como sus limitaciones, relacionadas principalmente con el préstamo interbibliotecario y los derechos de autor o copia.

Los defensores del acceso abierto argumentan que los derechos de autor son compatibles con el acceso abierto toda vez que los autores rara vez buscan el beneficio económico con la publicación de sus trabajos. Son los editores quienes ostentan el derecho de copia y buscan beneficiarse con el acceso, la distribución y la reproducción del trabajo de los científicos. 
A través del acceso abierto se pretende derribar las barreras impuestas por los estatutos o el copyright, los contratos o licencias e incluso el software y hardware.

\section{Calidad de la información y acceso abierto}

Aunque la aparición de la revista electrónica ofrece muchas ventajas y promete más y mejores servicios, presenta retos y amenazas con relación a la accesibilidad de la información. Las publicaciones periódicas de acceso abierto son publicaciones evaluadas por pares académicos y pueden ser consultadas y leídas en línea por todas las personas sin ningún costo. En muchos casos también pueden ser publicadas en forma impresa, y algunas, principalmente aquellas que emanan de departamentos universitarios o de quienes cuentan con subsidios sustanciales, no cobran por autores o por páginas (JISC 2005).

El cuidado de la calidad de la información es un aspecto en el que iniciativas y proyectos coinciden. Se hace hincapié en que las publicaciones de acceso libre no deben ser consideradas como un mecanismo que facilite la auto-publicación o como una forma de que los autores evadan la evaluación de sus pares. Sobre la calidad de las publicaciones de acceso libre el Joint Information Systems Comité introduce el factor de impacto del siguiente modo:

[...] Muchos de los títulos de Acceso Abierto tienen un factor de impacto alto y son indexados por el Institute for Scientific Information para su Web of Knowledge/Web of Science service. En junio de 2004 se tenían en ese servicio 239 títulos de Acceso abierto. [...] Existe evidencia creciente que muestra que los artículos de investigación que han sido incorporados a los archivos de acceso abierto son más citados que otros que no lo están. Si se evalúan todas las áreas del conocimiento, el número de citas se ha duplicado cuando menos. En algunas áreas específicas se han incrementado aún más las citas. Esta modalidad de acceso abierto significa que la investigación tiene un impacto mayor que el que tenía anteriormente. Sin embargo el ciclo de la información, en donde el trabajo es publicado, leído, citado y otros investigadores construyen sobre ese conocimiento, se ve acelerado cuando los resultados están a la disposición en acceso abierto (JISC 2005).

Sin embargo, de todas las aseveraciones anteriores no se proporciona un dato duro que permita evaluar lo que se sostiene. No se indica cómo se probó el incremento en uso o en citas, no se sabe en qué áreas específicas se incrementó más la consulta, tampoco se proporciona información que sustente el comentario de que la investigación publicada en acceso abierto tiene mayor impacto. 
Contrariamente a lo anterior, un experimento sobre acceso abierto que se llevó a cabo a lo largo de un año y que fue publicado por la universidad de Oxford ha concluido que el modelo de acceso abierto en la modalidad del autor-paga tuvo un nivel de aceptación "generalmente bajo", por lo que durante ese periodo el modelo demostró no ser suficiente por sí mismo para apoyar los requerimientos de diversas comunidades de investigación (Demand for open access 2006, 1).

\section{Asociaciones bibliotecarias y acceso abierto}

Otro documento importante para entender el fenómeno del Acceso Abierto es el emanado por la Association of College and Research Libraries (Asociación de Bibliotecas Universitarias y de Investigación de los Estados Unidos, ACRL por sus siglas en inglés) titulado Principios y Estrategias para Reformar la Comunicación Erudita.

El documento principia por definir lo que la ACRL entiende por comunicación erudita:

... es el sistema a través del cual se crean escritos de investigación y otros trabajos doctos, que se evalúan por calidad [sic], se diseminan entre la comunidad académica y se preservan para el futuro ( ACRL 2003,155).

Se caracteriza después el problema que representa la concentración de las publicaciones en unos cuantos editores y el incremento de los precios más allá de las posibilidades de las bibliotecas, lo que las ha obligado a recortar suscripciones o bien a reducir la adquisición de obras monográficas:

[...] la industria de publicaciones de revistas se ha consolidado cada vez más y está ahora dominada por un pequeño número de conglomerados internacionales. Los precios de las series eruditas han sido elevados con tasas que superan de sobra la inflación general de la economía y también por encima de las tasas de incremento de los presupuestos de las bibliotecas (ACRL 2003,156).

Esto significa que la antigua actividad de préstamo bibliotecario, que era regida por la ley de derechos de autor, ahora se pacta mediante un contrato que depende de la habilidad que tenga el bibliotecario y su cuerpo legal para permitir el acceso de otras personas a la información adquirida. Se sufre en la actualidad, y en casi todos los países, la embestida de parte de aquellos que proponen disminuir el acceso público al ampliar el plazo de los derechos que protegen a los autores, con lo cual se afecta de forma significativa lo que se 
ha denominado el uso justo de la información, particularmente de la digital. Cuando las relaciones entre los productores de bases de datos y los productores de textos completos se modifican, también se modifican los servicios que las bibliotecas les ofrecen a sus usuarios.

La ACRL desea establecer un sistema de comunicación científica más receptivo a las necesidades del mundo académico, que refleje la naturaleza del saber y la investigación como bien público (ACRL 2003,157).

La iniciativa de la ACRL contempla muchos elementos que no son considerados por el documento de Budapest ni por el de Berlín; tiene además un tono más mesurado, y no parece un llamado al combate sino que pretende conciliar las opiniones entre los distintos actores (académicos, editores, agregadores de valor, bibliotecas y usuarios).

La Federación Internacional de Asociaciones e Instituciones Bibliotecarias (IFLA) también ha contribuido a definir lo que es el Acceso Abierto, así el 5 de diciembre de 2003 dio a conocer el documento denominado IFLA statement on open access to scholarly literature and research documentation (Declaración de IFLA sobre el acceso abierto a la literatura académica y de investigación). En este documento la IFLA presenta una serie de consideraciones sobre la comunicación vía archivos abiertos, donde afirma que el amplio acceso a la literatura y la documentación producidas por la investigación es vital para entender nuestro mundo e identificar soluciones a los retos globales, particularmente para reducir las desigualdades en el acceso a la información.

La definición sobre acceso abierto adoptada por la IFLA se basa en el documento Position statement by the Wellcome Trust in support of open access publishing (Declaración del Wellcome Trust en apoyo a la publicación de acceso abierto) que contiene la definición a la que llegaron los delegados que asistieron a la reunión que el Howard Medical Institute (Instituto Médico Howard) convocó en Julio de 2003.

Se ha pensado que el esquema de acceso abierto ayuda a las bibliotecas, principalmente a aquellas que cuentan con menos recursos, a que sus usuarios tengan la posibilidad de utilizar materiales de calidad e incluso la posibilidad de bajar la información, así como a formar sus propios archivos y a garantizarles a los usuarios que esos materiales se encontrarán a su disposición siempre.

\section{Conclusiones}

En un ejercicio de síntesis se puede decir que el movimiento a favor del acceso libre a las publicaciones académicas y científicas es resultado del alto costo de las suscripciones que ha restringido el acceso a la información. La industria editorial, dio cabida a monopolios de la información, construyó enormes 
barreras económicas imposibles de sortear, y ha creado con ello una paradoja que implica que la comunidad generadora del conocimiento no puede acceder a la información que genera. Por tal motivo, diversos sectores académicos, científicos y profesionales se han reunido convocados por el paradigma del acceso abierto.

Las iniciativas, declaraciones y proyectos que se vinculan con el acceso abierto favorecen el acceso libre e irrestricto de los trabajos publicados por la comunidad científica, y discuten asuntos relacionados con el costo de la información, la tecnología, la interoperabilidad, los derechos de autor y la calidad de la información, e incluso han dado origen a acciones concretas de acceso libre.

En las iniciativas y declaraciones que se han presentado a lo largo de este trabajo se pueden observar los aspectos comunes que le dan a este modelo sus rasgos característicos.

El acceso abierto se basa en:

1. Las TIC como medio de diseminación.

2. El derecho de los autores sobre sus creaciones; es decir, se reconocen y respetan las leyes de copyright.

3. La colaboración entre quienes generan conocimiento y quienes poseen datos y/o información.

4. El desarrollo de la infraestructura necesaria y de los mecanismos estandarizados que garanticen la interoperatividad.

5. La gratuidad de la información y la derogación de barreras económicas para acceder a la información.

6. Información de calidad y libre de censura.

7. La eliminación, tanto como sea posible, de intermediarios.

8. La búsqueda de nuevas formas de financiamiento.

Lo que se quiere es lograr:

1. Mejorar el sistema de comunicación científica y académica mundial.

2. Asegurar la disponibilidad y preservación de la información.

3. Ampliar la visibilidad de los autores.

4. Diseminar la información y el conocimiento.

5. Distribuir mundialmente literatura científica de calidad sin restricciones y gratuitamente.

6. Reducir los costos de la información.

Se ha demostrado que el acceso abierto es posible a pesar de que se enfrenta, por su naturaleza, con los intereses que dominan la industria editorial. 
En la constitución de las diferentes iniciativas de acceso abierto de los que ha dado cuenta este documento se han reunido los diferentes actores involucrados en el ciclo de la comunicación científica: científicos, asociaciones científicas, instituciones académicas que patrocinan y/o generan investigación, editores académicos y comerciales, proveedores y/o agregadores de valor y bibliotecas y bibliotecarios. Sin embargo, a pesar del argumento generalizado de que son los científicos quienes usan la información científica, un sector de los usuarios no ha participado en estas iniciativas. Es el caso del usuario común, aquél que utiliza la información como medio de mejoramiento personal o formación académica, y quizás valga la pena reflexionar sobre su participación en el acceso abierto.

\section{REFERENCIAS}

ACRL 2003, "Principios y estrategias para reformar la comunicación erudita”, en Geotrópico [En línea], vol. 1, no. 2, pp. 155-159, disponible en: http://www.geotropico.org/1_2_Documentos_ACRL_ Principios.html [Agosto 2, 2006].

Anderson, R 2004, Open access in the real world : confronting economic and legal reality, en CERL News [en línea], vol. 65 no. 4, disponible en: http://www.ala.org/ala/acrl/acrlpubs/crlnews/ backissues2004/april04/openaccess.htm [Agosto 2, 2006].

Bethesda Statement on Open Access Publishing, [en línea] 2003 disponible en http://www.earlham.edu/ peters/fos/bethesda.html [Noviembre 28 2006]

BOAI 2002, Budapest open access initiative, [dn línea], disponible en http://www.soros.org/openaccess/read.shtml [Junio 20 2006].

Corrado, E M 2005, "The importance of open access, open source, and open standards for libraries", en Issues in Science and Technology Librarianship, [en línea] disponible en http://www.istl. org/05-spring/article2.html [Junio 19, 2006]

De Gennaro, R 1977, "Escalating journal prices: time to fight back", en American Libraries vol. 8 , no. 2, pp. 69-74.

Demand for open access 'low' (2006), Information World Review, [en línea], disponible en : http://www.iwr.co.uk/information-worldreview/news/2163737/demand-open-access-low [Septiembre 19, 2006]

DOAJ: directory of open access journals, [en línea] 2006, disponible en: http://www.doaj.org/ [Junio 20, 2006]

Federal Research Public Access Act of 2006, [en línea], disponible en: http://cornyn.senate.gov/doc_archive/05-02-2006_COE06461_ xml.pdf [Junio 20, 2006]. 
Goodman, D 2004, “The criteria for open access", en Digital Library of Information Science and Technology, [en línea], disponible en http://dlist.sir.arizona.edu/798/ [Agosto 1, 2006].

IFLA 2003, IFLA statement on open access to scholarly literature and research documentation, [en línea], disponible en: http://www.ifla. org/V/cdoc/open-access04.html [Julio 1, 2006]

Jaschik, S 2006, Rallying behind open access, [En línea], Inside Higher Ed, Washington D.C., disponible en: http://www.insidehighered.com/news/2006/07/28/provosts [Julio 2, 2006]

JISC 2005, Open access, [en línea], disponible en http://www.jisc. ac.uk/index.cfm? name=pub_openaccess [Junio 26, 2006]

Melero, R 2005, "Acceso abierto a las publicaciones científicas : definición, recursos, copyright e impacto”, en El Profesional de la Información, [en línea] vol. 14, no. 4, Disponible en: http://eprints. rclis.org/archive/00004371/01/EPI-rmelero.pdf [Agosto 11, 2006]

An open letter to the higher education community, [en línea], disponible en: http://www.cic.uiuc.edu/groups/CICMembers/archive/documents/FRPAAletterFinal7-24-06.pdf [Agosto 2, 2006]

Sociedad Max Plank 2003a, Berlin declaration on open access to knowledge in the sciences and humanities, [en línea], disponible en: http://www.zim.mpg.de/openaccess-berlin/berlindeclaration.ht$\mathrm{ml}$ [Junio 20, 2006].

Sociedad Max Plank 2003b, "La declaración de Berlín sobre acceso abierto”, Geotrópico, [en línea], vol. 1, no. 2, pp. 152-154, disponible en: http://www.geotropico.org/1_2_Documentos_Berlin.html [Julio 6, 2006].

Suber, P 2002, "Open access to the scientific journal literature", en Journal of Biology, [en línea], disponible en: http://jbiol.com/content/pdf/1475-4924-1-3.pdf [Septiembre 6, 2006].

Suber, P 2004, A very brief introduction to open access, [en línea], disponible en http://www.earlham.edu/ peters/fos/brief.htm [Septiembre 18, 2006].

Suber, P 2006, Open access overview, [en línea], disponible en http:// www.earlham.edu/ peters/fos/overview.htm [Septiembre 18, 2006].

Triska, R, Café, L 2001, "Arquivos abertos subproducto da biblioteca digital brasileira”, en Ciencia da Informacao, [en línea], vol. 30, no. 3, pp. 92-96, disponible en: http://www.ibict.br/cienciadainformacao/viewarticle.php?id=241\&layout=abstract [Agosto 1, 2006].

Willinsky, J 2003, The Nine Flavours of Open Access Scholarly Publishing, en Journal of Postgraduate Medicine, [en línea], vol. 49, pp. 263-267, disponible en http://www.jpgmonline.com/article. asp issn $=0022-3859$; year $=2003$; volume $=49$; issue $=3$; spage $=263$; epage $=267$; aulast $=$ Willinsky [Septiembre 6, 2004] . 


\section{OBRAS CONSUlTADAS}

Bailey, J C 2005, Open Access bibliography, ARL, Washington.

Jaschik, S 2006, In whose interest?, [en línea], Inside Higher Ed, Washington D.C., Disponible en: http://www.insidehighered. com/news/2006/06/15/open [Julio 2, 2006] , 2006, New model for scholarly publishing, [en linea], Inside Higher Ed, Washington D.C., disponible en: http://www.insidehighered.com/news/2006/07/14/rice [Julio 30, 2006].

Kwasik, H, Pauline O F 2005, "Open access and scholarly communication : a selection of key web sites", en Issues in Science and Technology Librarianship, [en línea], disponible en: http://www.istl. org/05-summer/internet.html [Junio 20,2006].

Lagoze, C, Sompel, H 2001, The open archives initiative : building a low-barrier interoperability framework, [en línea], disponible en http://www.openarchives.org/documents/jcdl2001-oai.pdf [Agosto 9, 2006].

Miller, P 2000, Interoperability : what is it and why should I want it?, en Ariadne, [en línea], 24 June. disponible en: http://www.ariadne.ac.uk/issue24/interoperability/ [Agosto 9, 2006].

Rucinque, H F, Durango-Vertel, J 2003, "Revistas electrónicas, autoarchivo y acceso abierto : retos nuevos y alternativas para la publicación geográfica en el mundo tropical”, en Geotrópico, [en línea], vol. 1, no. 1, pp. 4-10, disponible en: http://www.geotropico.org/ Editorial_1_1.pdf [Julio 5, 2006].

\section{Sitios DE INTERÉS}

Directory of Open Access Journals http://www.doaj.org/

Google académico http://www.scholar.google.com.br

Open Archives initiative http://www.openarchives.org/index.html 